This is a postprint version of the following published document:

Mikeš, F., González-Benito, J., \& Baselga, J. (2001).

Monitoring of curing process by fluorescence technique. Fluorescence probe and label based on 5dimethylaminonaphthalene-1-sulfonamide derivatives (DNS). Journal of Macromolecular Science, Part B, 40 (3-4), pp. 405-428.

DOI: $10.1081 / \mathrm{MB}-100106167$

(C) Taylor \& Francis, 2001 


\title{
Monitoring of curing process by fluorescence technique. Fluorescence probe and label based on 5-dimethylaminonaphthalene-1-sulfonamide derivatives (DNS)
}

\author{
František Mikeš - Javier González Benito - Juan Baselga
}

\begin{abstract}
The curing reaction of glycidyl ether bisphenol A (DGEBA) with $n$-butyl amine and/or $N$-methylethylenediamine was monitored by fluorescence spectroscopy. 5-Dimethylaminonaphthalene-1-sulfonamide (DNS) fluorophore was used as a probe and/or label. Fourier transform infrared (FTIR) analysis revealed that the rate constant for the addition reaction of the primary amino group hydrogen of $n$-butylamine to the epoxide ring is more than four times larger than that arising from a secondary amine. Significant differences have been observed between the fluorescence behavior of the DNS as a probe and label, especially in the system DGEBA- $n$-butyl amine. Integrated fluorescence intensity for the DNS label, in contrast to the DNS probe, indicates the most important changes in chemical transformations of this reaction mixture (the onset of tertiary amino groups and maximum concentration of secondary amino groups).

Similarly, the dependence of the half-bandwidth on the epoxy groups conversion for the DNS label shows these stages of the curing reaction as well. In the system DGEBA- $N$-methylethylenediamine, the reactivity of the secondary amino group hydrogen is higher than that of the primary amino group. A change in slope of the dependence of integrated fluorescence intensity on epoxy group conversion clearly indicates the gel point and entry of the system into the glassy state. The DNS probe does not sense any of these changes. From the emission spectra of the DNS probe and/or label, the average value $<\mathrm{v}>=\Sigma I_{F}(v) v / \Sigma I_{F}(v)$ of the emission band position has been correlated with the epoxy group conversion determined by FTIR. Smooth dependencies were obtained in all cases. This enables one to monitor on line and in real time the epoxy group conversion.
\end{abstract}

\section{Keywords}

Bisphenol A, Dansyl probe and label, Diglycidyl ether, Epoxide curing, Fluorescence monitoring, FTIR analysis, Gel point, Glassy state

\section{INTRODUCTION}

The structural properties of advanced composites based on epoxide resins are known to depend strongly on the extent 
of cure. Several physicochemical methods have been applied for characterization of cure phenomena in epoxies. To optimize the curing cycle, on-line and real-time methods are preferentially desired. Only several methods can be technically adopted for this purpose. Of these methods, undoubtedly the most promising is fluorescence spectroscopy 1-11. Fluorescence spectroscopy has been gaining increasing attention for these applications since the mid-1980s due to its sensitivity, selectivity, and nondestructive characteristics.

Literature covering this subject includes, in principle, intrinsic and extrinsic fluorophore techniques $4-11$. The molecular environment of a small molecule can be monitored by means of its fluorescence characteristics; this has been widely used in the past. Such molecules are usually designed to examine specific properties of their immediate environment or microenvironment by means of a shift in the wavenumber of their emission maximum or a change in emission intensity and the half-bandwidth. Probes that monitor the polarity $12-13$ and those that monitor the rigidity of the matrix [14] in which they reside are well known. Other options are probes or labels to monitor changes in the

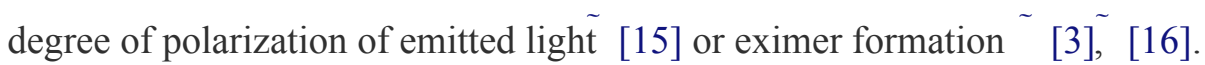

To the best of our knowledge, there has not been any contribution comparing the use of the same fluorophore as a probe and as a label to monitor the curing process in epoxies. The main objectives of this research, in comparison with existing studies carried out in the past, can be summarized as follows:

1. To follow kinetics of the curing reaction for the epoxide-amine systems diglycidyl ether bisphenol A (DGEBA)- $n$-butylamine and/or $\quad N$-methyl-ethylenediamine and to determine the reaction rate constants for the system DGEBA- $n$-butylamine.

2. To correlate the observed changes in the fluorescence characteristics for 5-dimethylaminonaphthalene-1sulfonamide (DNS) label and DNS probe (integrated fluorescence intensity, position of the emission maximum, and half-bandwidth) with the extent of the epoxy group conversion.

3. We would like to demonstrate that the process of curing of epoxide-amine formulations monitored by changes in fluorescence characteristics can be correlated with the most important characteristics of the curing process, that is, the onset of formation of tertiary amino groups, maximum concentration of secondary amino groups, the gel point, and the onset of the glassy state.

4. To show that, in some cases, the response of the DNS fluorophore used as a probe or label may be entirely different.

5. Moreover, we would like to show that the average value of the emission band position defined by $\Sigma I_{F}$

$(v) v / \Sigma I_{F}(v)$ can be successfully correlated with epoxy group conversion.

\section{EXPERIMENTAL}




\section{Preparation of Probe and Label Precursor}

Preparation of 5-dimethylaminonaphthalene-1-( $\quad N$-(di- $n$-butyl)(sulfonamide (DNSd-Bu) (probe) and DNS label precursor 5-dimethylaminonaphthalene-1-(2-aminoethyl)sulfonamide (DNS EDA) used for labeling of the diepoxide (DGEBA) has already been described 17-18 .

Diglycidyl ether bisphenol A (DGEBA) (molecular weight $348 \mathrm{~g} / \mathrm{mol}$ ) (Aldrich, Madrid, Spain) was purified as discussed elsewhere [17]. N-Methylethylenediamine (N-MEDA) and $n$-butylamine (n-BuA) were Aldrich products. Both amines were boiled under reflux over potassium hydroxide and rectified under nitrogen atmosphere. 5Dimethylaminonaphthalene-1-sulfonyl chloride (DNS chloride) (Lancaster Synthesis, Ltd., Bischheim, France) was used as received.

\section{Labeling of the Epoxide Component}

Labeling of the epoxide component (DGEBA) by reaction with the reactive DNS label precursor DNS EDA was carried out by heating a mixture of both components under stirring at $60^{\circ} \mathrm{C}$ for $6 \mathrm{~h}$ as described elsewhere [17].

\section{Methods}

\section{Differential Scanning Calorimetry}

A Perkin Elmer DSC-7 (Perkin Elmer Corp., Norwalk, CT) differential scanning calorimeter (DSC) equipped with a standard Perkin Elmer cooling unit was used for measurements. Samples weighing 10-15 mg were measured. DSC was used to determine the glass transition temperature for the stoichiometric mixtures DGEBA- $n$-butylamine and/or $\mathrm{N}$-methylethylenediamine. Curing experiments were carried out in the DSC apparatus at desired temperatures and for desired reaction times. Ultimate curing conversion was achieved by dynamic scan $\left(30^{\circ} \mathrm{C}-170^{\circ} \mathrm{C}\right)$ at a scanning rate of $5^{\circ} \mathrm{C} / \mathrm{min}$. The glass transition temperature was evaluated by a computer program supplied by PE.

\section{Fourier Transform Infrared Spectroscopy}

For Fourier transform infrared spectroscopy (FTIR), a Perkin Elmer GX FTIR (Perkin Elmer, Ltd., Beaconsfield Bucks, England) spectrometer was used to monitor the rate of disappearance of the epoxy ring and primary amino group. All spectra were collected in the near infrared (IR) region $\left(7000-4000 \mathrm{~cm}^{-1}\right)$. Each spectrum was obtained, depending on rate of curing reaction, by averaging 4 to 20 scans at $4 \mathrm{~cm}^{-1}$ resolution with scanning rate (OPD) of $0.2 \mathrm{~cm} / \mathrm{s}$. Measurements were carried out at $40^{\circ} \mathrm{C}$ and $60^{\circ} \mathrm{C}$ using a SPECAC temperature controller. The epoxide formulations were cured in disposable cells made from the microscope glass slide plates cut to fit the diameter of the cell holder (Ø $25 \mathrm{~mm}$ ) with an optical path of 0.7-1.0 mm determined by the thickness of a Teflon spacer. The values of absorbance and integrated absorbance corresponding to ultimate curing were obtained after curing at 
$180^{\circ} \mathrm{C}$ (DGEBA- $n$-butylamine) or $200^{\circ} \mathrm{C}$ (DGEBA- $N$-methylethylenediamine) for at least $2 \mathrm{~h}$.

The main spectral bands of interest in the cure reaction and their assignment are in general agreement with those previously reported 19-21. The main features in the spectra are 1 a decrease in the epoxy band at around $4530 \mathrm{~cm}^{-1}$, 2 a decrease in the primary amine groups at $4938 \mathrm{~cm}^{-1}$, and 3 an increase in the hydroxyl bands in the region around $4800 \mathrm{~cm}^{-1}$. The most significant feature is the appearance of the isosbestic point between the epoxy and hydroxyl group bands. This indicates that there is a direct relationship between the disappearance of the epoxy and the appearance of the hydroxyl group, and that Beer's law is obeyed over a wide range of conversion [20]. It is therefore possible to use band height alone as a measure of epoxy groups concentration. At the same time, integration of the epoxy group band was carried out.

The analysis consists of measuring the absorbance and integrated absorbance at two specified wavenumbers-one corresponding to the changing epoxy group peak and the other to an invariant $\mathrm{CH}$ band. In each set of spectra, a band appearing invariant was chosen. Surprisingly, very good agreement has been found between these two procedures.

\section{Ultraviolet/Visible Spectroscopy}

All spectra were taken on a Perkin Elmer ultraviolet/visible (UV/Vis) spectrometer Lambda 14P (Perkin Elmer GmBH, Uberlingen, Germany). Absorption UV/Vis spectra of the components and epoxide resin formulations with and without fluorophore were measured in home-made cells from microscope glass slide plates or spectrometric grade poly(methyl methacrylate). Details have been published elsewhere [17].

\section{Fluorescence Measurements}

Steady-state fluorescence spectra were taken on a Perkin Elmer luminescence spectrometer LS 50B (Perkin Elmer, Ltd.). The samples of the neat epoxide resin formulations either labeled (probe or label) or nonlabeled were measured in disposable thermostated cells made from microscope slide glass plates. Front-face $\left(60^{\circ} / 30^{\circ}\right)$ illumination geometry was used. Time-dependent changes in emission spectra with progress of the curing reaction were recorded using the same fluorimeter provided with a home-made obey program. From any particular emission spectrum, the integrated fluorescence intensity, emission maximum, half-bandwidth, and average value of emission band position (the first moment of the emission band) [22] $(\langle v\rangle)$ of the DNS emission band defined by $\Sigma I_{F}$ $(v) v / \Sigma I_{F}(v)$ were obtained.

\section{RESULTS AND DISCUSSION}




\section{Ultraviolet/Visible and Fluorescence Spectra}

The fluorescence label and probe used in this study of the systems DGEBA- $n$-butylamine and/or $N$ methylethylenediamine cover the excitation spectral range 320-380 nm. Based on the absorption spectra of the labeled and unlabeled epoxide-amine reaction mixtures, the observed emission (excitation $350 \mathrm{~nm}$ ) in curing experiments represents the emission of the DNS fluorophore only, as discussed in our previous communication [17]. It should be pointed out that, for front-face excitation, the emission intensity depends only on the ratio of the DNS absorbance to total absorbance $A_{T}$ of the reaction mixture at the excitation wavelength condition for front-face fluorometry $A_{T} t \quad 1$ where $t$ is the cell thickness $\quad$ [23]. This condition was fulfilled in all experiments.

Since in our case the total absorbance at the excitation wavelength $(350 \mathrm{~nm})$ was equal or very close to the DNS absorbance, potential changes in DNS absorbance with increasing conversion would not influence the intensity of the DNS emission. All changes in fluorescence intensity of the DNS fluorophore are caused by changes in environment of the DNS structural units. The reabsorption of the emitted radiation in spite of rather high optical density did not take place owing to a large Stoke's shift of the DNS fluorophore in these reaction media. For fluorescence monitoring of the curing progress in the stoichiometric mixtures DGEBA- $n$-butylamine and/or $N$ methylethylenediamine, the concentration of DNS fluorophore (label and/or probe) was $5.088 \times 10^{-3}$ and $5.391 \times$ $10^{-3} \mathrm{~mol} / 1000 \mathrm{~g}$ of reaction mixture, respectively.

\section{Extent of Reaction and Reaction Kinetics of Diepoxide with Primary Amine and/or Amine-Containing Primary and Secondary Amino Groups}

Satisfactory interpretation of fluorescence data for epoxide systems requires knowledge not only of the cure time dependence of the epoxy group conversion, but also knowledge of the other physicochemical parameters characterizing the curing systems (i.e., dependence of the glass transition temperature on epoxy group conversion, reaction kinetics data, and conversion of epoxy groups at the gel point). In this article, two very different epoxideamine systems were studied: DGEBA- $n$-butylamine, which gave a linear polyaddition product, and the cross-linking DGEBA- $N$-methylethylenediamine system.

\section{DGEBA- $n$-Butylamine}

The obtained experimental data for the system DGEBA- $\quad n$-butylamine were analyzed according to kinetic equation [17], [24], assuming simultaneous autocatalyzed and catalyzed mechanisms for stoichiometric reaction mixture:

$$
d \alpha / d t /(1-\alpha)^{2}\left(1+2 a_{2} \Delta n /\left(2 a_{1}+a_{2}\right)\right)=\left(k_{1} e_{0}^{2} / 2\right) \alpha+k_{1}^{\prime} e_{0} c_{0} / 2
$$

where $a_{0}$ and $e_{0}$ are the initial concentrations of primary amine and epoxide, respectively; $\alpha$ is the epoxy group conversion; and $k_{1}$ and $k_{1}^{\prime}$ are reaction rate constants for autocatalyzed and catalyzed reactions of epoxide with primary amine, respectively. Concentrations of the primary and secondary amine at epoxy group conversion $\alpha$ are $a$ 
1 and $a_{2}$, respectively. $\quad c_{0}$ represents the concentration of catalyst, and $\Delta n$ is defined by the relation of Eq. 2 :

$$
k_{2} / k_{1}=k_{2}^{\prime} / k_{1}^{\prime}=n=1 / 2+\Delta n
$$

where $k_{2}$ and $k_{2}^{\prime}$ are reaction rate constants of autocatalyzed and catalyzed reactions of epoxide with secondary amine, respectively. FTIR analysis provides experimental data over the whole range of conversion, and the left-hand side of Eq. 1 can be plotted against $\alpha$. The concentrations of the epoxy groups and primary amine $\left(a_{1}\right)$ were directly evaluated from the experimental data, and the concentrations of secondary and tertiary amines were determined from the mass balances. The concentration of secondary amine $a_{2}$ is equal to

$$
a_{2}=e_{0}(\beta-\alpha)
$$

and the concentration of tertiary amine is given by Eq.

$$
a_{3}=e_{0}(\alpha-\beta / 2)
$$

where $\beta$ is the conversion of primary amine groups, $\left(\left(a^{\prime}{ }_{1}-a_{1}\right) / a^{\prime}{ }_{1}\right)$.

The ratio of the rate constants $\quad k_{2} / k_{1}$ was evaluated according to the method of Paz-Abuin et al. [25].

In the addition reaction of primary amine hydrogen of $\quad n$-butylamine to an epoxide ring, a secondary amine is formed. The secondary amine further reacts with the epoxy group, forming, in this case, a linear polymer with short $n$-butyl side chains. The dependence of epoxy group conversion on cure time at $40^{\circ} \mathrm{C}$ is shown in Fig. 1a. From Fig. 1b, one can assume that the reaction rate constant $k_{1}$ for reaction of the primary amine hydrogen of $n$-butylamine is much larger than for the secondary amine $k_{2}$. The ratio of the rate constants $k_{2} / k_{1}$ determined from Fig. $1_{b}$ is equal to 0.23 . In this case, for a long time, the concentration of secondary amine will be built up faster than it is depleted, and the maximum concentration of secondary amine will be relatively high. The change from primary amine to secondary amine occurs relatively quickly; the change from secondary amine to tertiary amine occurs slowly. For reaction times longer than $180 \mathrm{~min}$, the curves for secondary and tertiary amine species are essentially identical to those for a simple first-order process.

Figure 1. The dependence of (a) the epoxy groups conversion $\alpha$, (b) the primary $a_{1}$, secondary $a_{2}$, and tertiary $a_{3}$ amine concentrations, and (c) the integrated fluorescence intensity (Integ. Fluor. Int.) for the DNS label and probe on cure time $t$ for the stoichiometric reaction mixture DGEBA- $n$-butylamine. Curing temperature $40^{\circ} \mathrm{C}$. 


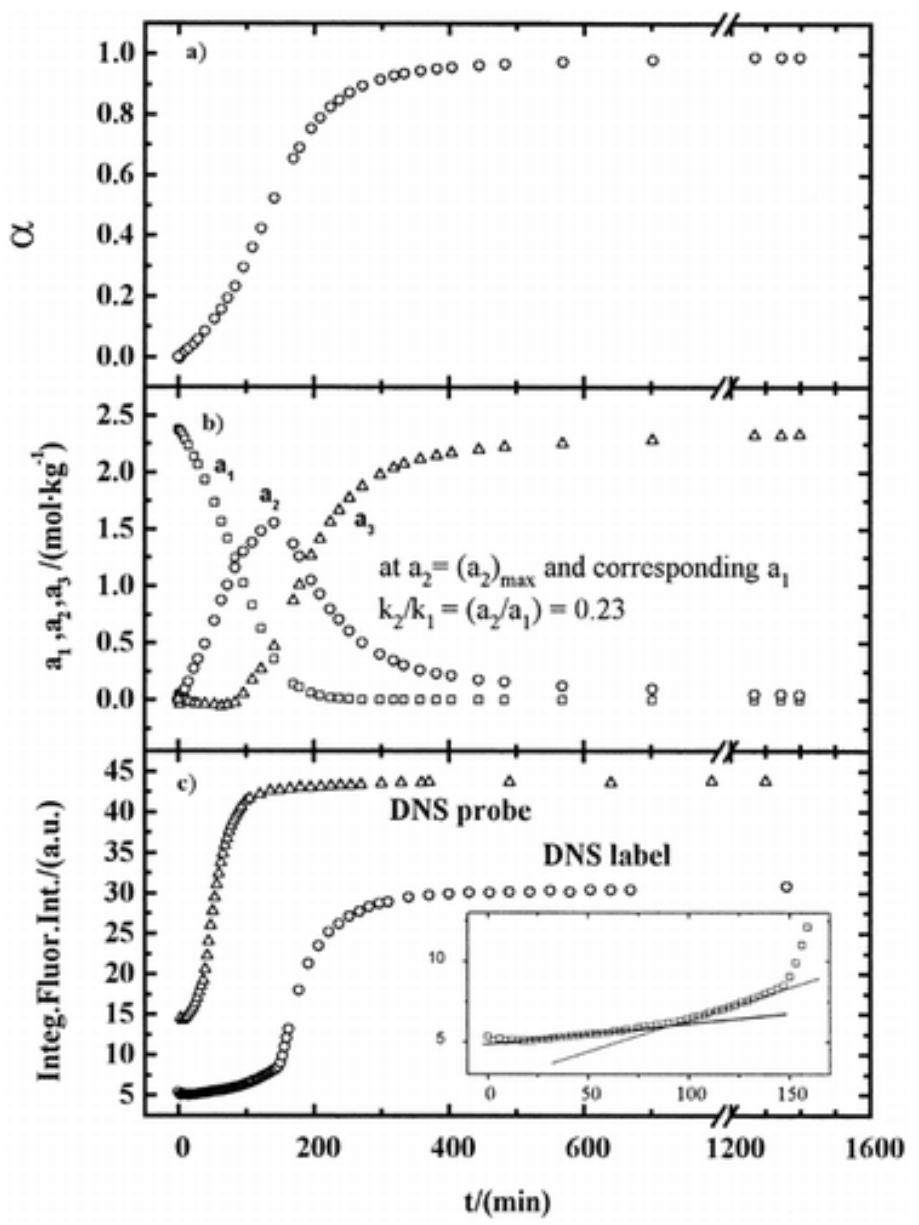

The glass transition temperatures (Fig. 2a) of DGEBA- $n$-butylamine reaction mixtures, independent of conversion, are always lower than $T$ cure $=40^{\circ} \mathrm{C}$. Therefore, the curing proceeds at the cure temperature of $40^{\circ} \mathrm{C}$ in the rubbery state. The reduced reaction rate according to Eq. 1 is plotted versus epoxy group conversion in Fig. 2 b. This dependence obeys a linear relationship up to $\alpha=0.55$. Deviation from the linear dependence was supposed to be the cause for the reaction of phenyl glycidyl ether with $N$-butylamine [24] by the existence of traces of impurities accelerating the reaction in the system. Another reason, and more probable, might be the fact that the proposed autoacceleration mechanism does not exactly describe the kinetic behavior of the system, especially at higher conversions.

Figure 2. The dependence of (a) the glass transition temperature $T_{\mathrm{g}}$, (b) the reduced reaction rate, and (c) normalized integrated fluorescence intensity (Norm. Integ. Fluor. Int.) for the DNS probe and label on the epoxy groups conversion $\alpha$ for the stoichiometric reaction mixture DGEBA- $n$-butylamine. Curing temperature $40^{\circ} \mathrm{C}$. 


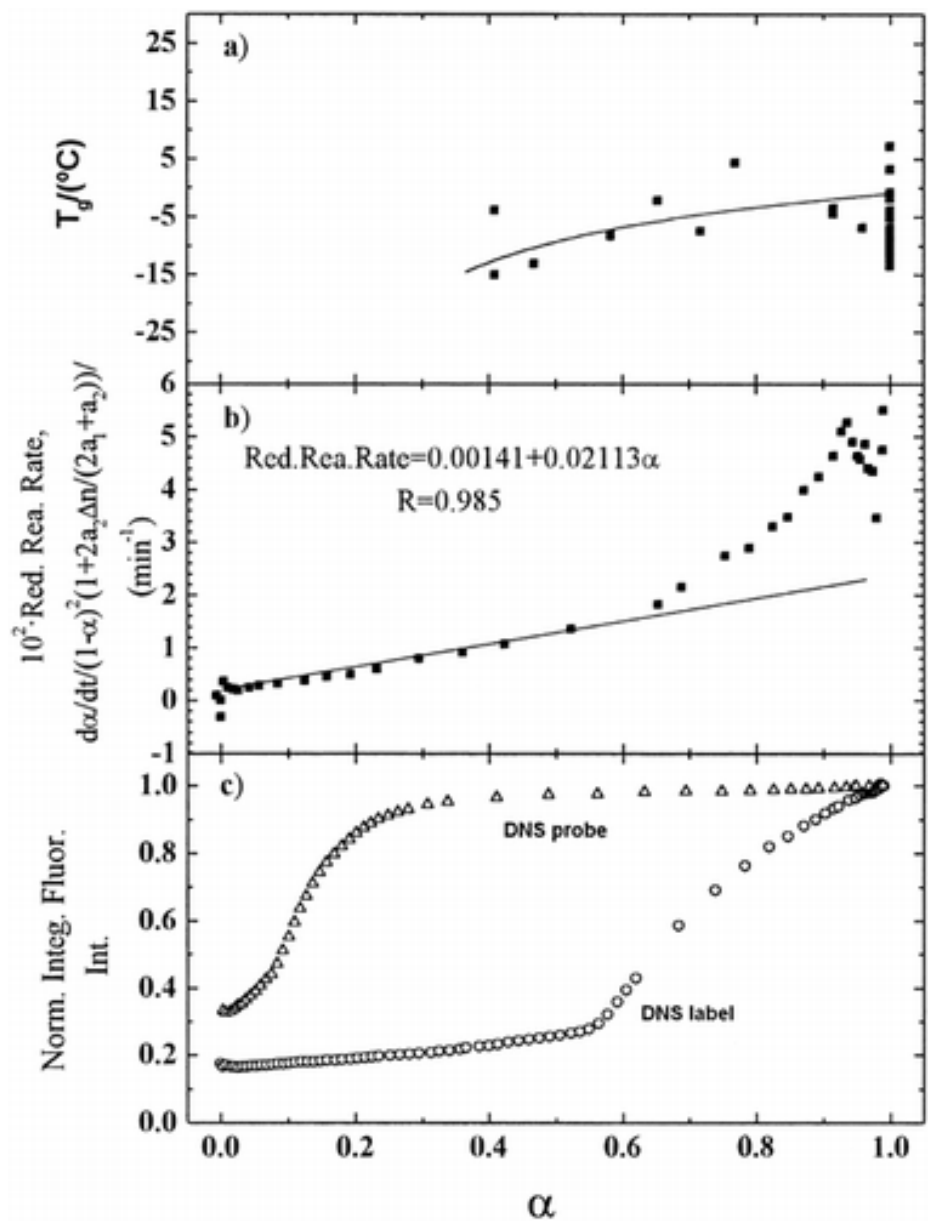

Evaluated reaction rate constants $\quad k_{1}$ and $k^{\prime}{ }_{1} c_{0}$ were $1.9 \times 10^{-3} \mathrm{~kg}^{2} / \mathrm{mol}^{2}-\mathrm{min}$ and $5.9 \times 10^{-4} \mathrm{~kg} / \mathrm{mol}-\mathrm{min}$ at $40^{\circ} \mathrm{C}$, respectively. The reaction rate constant $\mathrm{k} 2=4.3 \times 10^{-4} \mathrm{~kg}^{2} / \mathrm{mol}^{2}$-min at $40^{\circ} \mathrm{C}$. The reaction rate constant $k_{1}$ is more than four times larger than that of $k_{2}$. Figure $1 \mathrm{~b}$ shows quite clearly that tertiary amino structural units appear in the reaction mixture at $40^{\circ} \mathrm{C}$ at reaction time $90 \min \left(\begin{array}{ll}\alpha & 0.19\end{array}\right)$ but, owing to the larger value of $k_{1}$, the concentration of secondary amine structural units still increases and attains its maximum at $140 \min \left(\begin{array}{ll}\alpha & 0.52\end{array}\right)$. Therefore, the reaction mixture at the end of this time interval consists mostly of low molecular weight compounds formed by mono- and diaddition of $n$-butylamine to the epoxy groups of DGEBA, unreacted DGEBA, and very low concentrations of $n$-butylamine and oligomers.

With increasing reaction time ( $t>140 \mathrm{~min}$ ), the concentration of the secondary amine decreases very fast owing to the addition of secondary amine hydrogen (mono- and diaddition products) to the epoxide ring. This reaction is accompanied by a fast increase in the concentration of tertiary amine groups and, at the same time, by a sudden increase in molecular weight of the linear polymer. These changes in chemical composition of the reaction mixture can be seen in the curing experiment carried out with DNS-labeled DGEBA (Fig. 1c).

\section{DGEBA- $N$-Methylethylenediamine}

In Fig. 3, dependence of the epoxy group conversion on cure time at $40^{\circ} \mathrm{C}$ and $60^{\circ} \mathrm{C}$ is shown. It should be pointed 
out that this reaction mixture, in progress, contains two types of secondary amino groups: those formed by reaction of the epoxy group with the primary amino group and secondary amino group in $\mathrm{N}$-methylethylenediamine already present at the beginning of the curing reaction. Similarly, the overall content of tertiary amino groups consists of tertiary amino groups formed from the originally present primary amino groups and from secondary methyl amino groups.

Figure 3. The dependence of the epoxy groups conversion $\alpha$ on the cure time $t$ for the stoichiometric reaction mixture DGEBA- $N$-methylethylenediamine. Curing temperature $40^{\circ} \mathrm{C}$ and $60^{\circ} \mathrm{C}$.

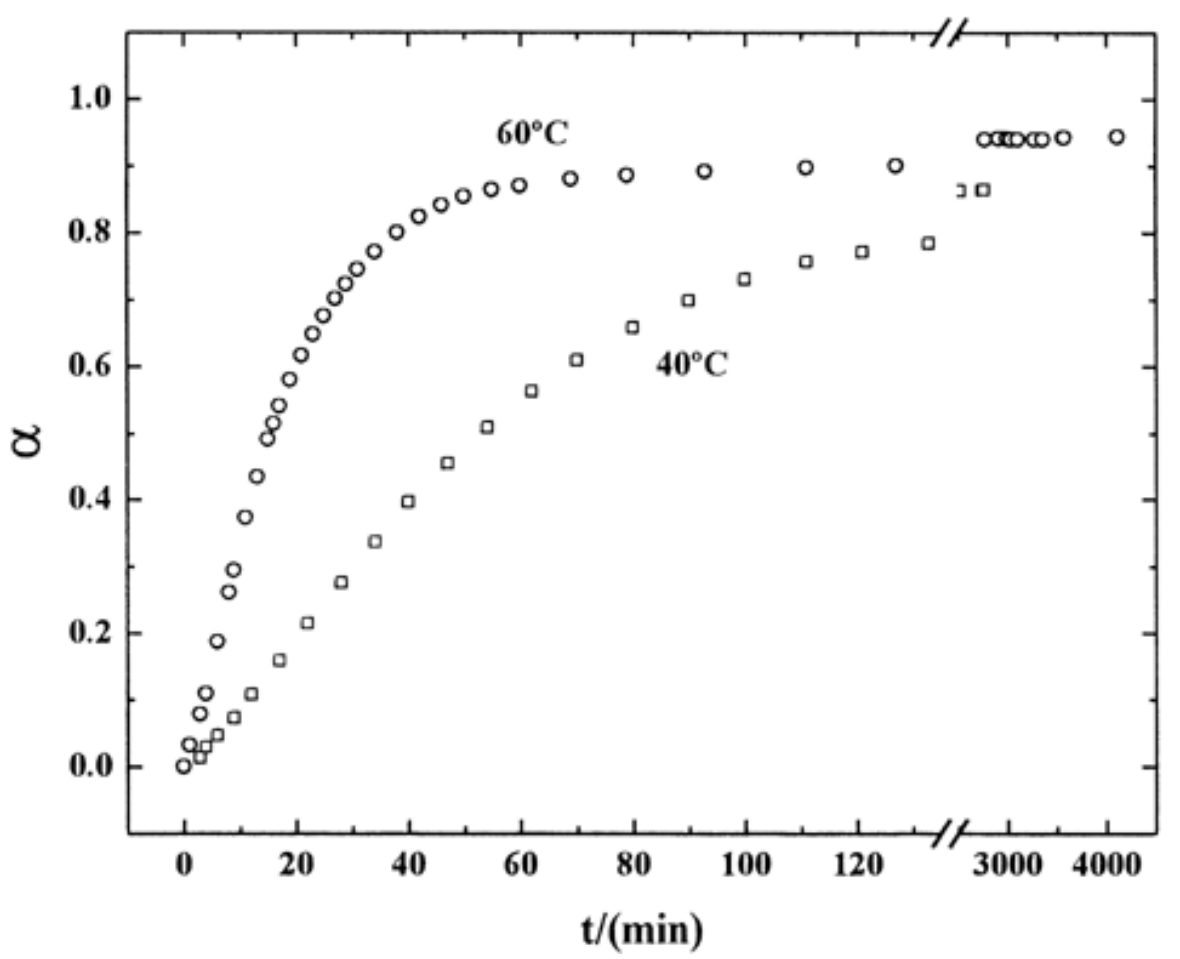

Let $e, a_{1}, a_{2}, a^{N}, a_{3}$, and $a^{N}{ }_{3}$ be the concentrations of the epoxy and primary, secondary, secondary

( $\mathrm{H} \mathrm{CH}_{3}$ ), tertiary, tertiary ( $\mathrm{N} \mathrm{CH}$ ) amino groups at reaction time $t$, respectively, and initial concentration of primary, secondary $\left(\mathrm{HCH}_{3}\right.$ ), and epoxy groups $\quad a^{0}{ }_{1}$, a ${ }^{N 0}$, and $e_{0}$, respectively. From the mass balances, the overall concentration of secondary $a{ }_{2}^{*}\left(a_{2}+a N_{2}\right)$ and tertiary $a{ }^{*}{ }_{3}\left(a_{3}+a N_{3}\right)$ amino groups can be derived:

$$
a_{2}^{\text {纱 }}=2 a_{1}^{0} \beta-e_{0} \alpha+a_{2}^{N 0}
$$

and

$$
a_{3}^{\omega}=e_{0} \alpha-a_{1}^{0} \beta
$$

The time dependence of the primary, overall secondary, and overall tertiary amino group concentrations is shown in Figs. $4 \mathrm{a}$ and $4 \mathrm{~b}$ for $40^{\circ} \mathrm{C}$ and $60^{\circ} \mathrm{C}$, respectively.

Figure 4. The time dependence of the primary $a_{1}$, overall secondary $a_{2}$, and overall tertiary $a_{3}$ amine concentrations for the stoichiometric reaction mixture DGEBA- $N$-methylethylenediamine. Curing temperature (a) 
$40^{\circ} \mathrm{C}$ and (b) $60^{\circ} \mathrm{C}$.
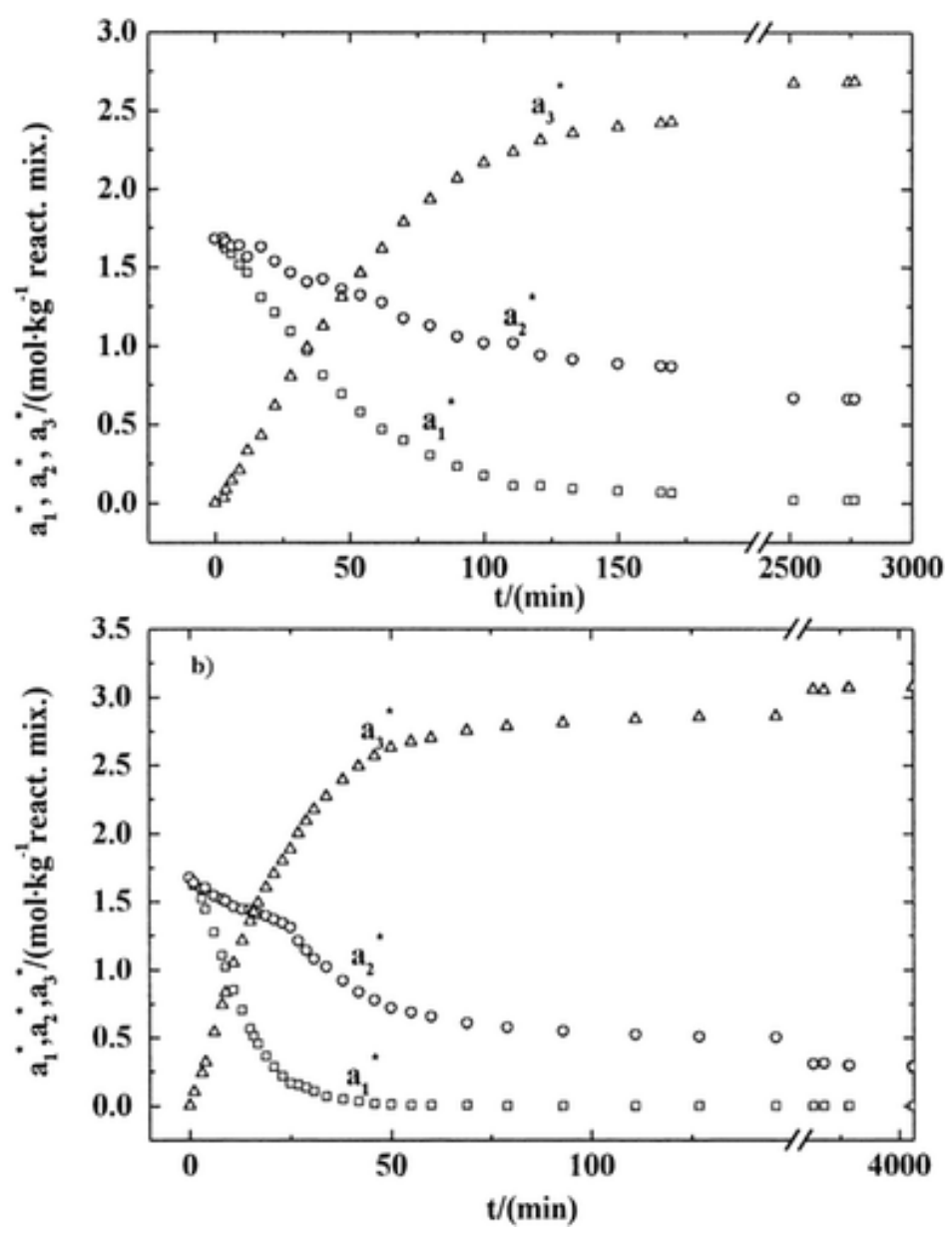

The most remarkable difference in comparison with similar dependencies for DGEBA-primary diamines is the immediate and fast increase in tertiary amino group concentration at the beginning of the reaction. This observation indicates that the reactivity of secondary amino group $\left(\mathrm{CH}_{3} \mathrm{H}\right.$ ) hydrogen with the epoxy group is higher than

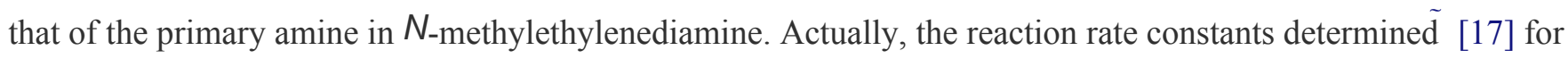
reaction of $N$, $N^{\prime}$-dimethylethylenediamine and ethylenediamine with epoxy groups are $k_{2}=33.1 \times 10^{-4} \mathrm{~kg}^{2} / \mathrm{mol}^{2}$ $\min$ and $k_{1}=16.5 \times 10^{-4} \mathrm{~kg}^{2} / \mathrm{mol}^{2}-\min , k_{2}=6.4 \times 10^{-4} \mathrm{~kg}^{2} / \mathrm{mol}^{2}-\min$ at $40^{\circ} \mathrm{C}$, respectively. The reaction rate constant for the reaction of secondary amine hydrogen with epoxy groups in $N, N^{\prime}$-dimethylethylenediamine is two times larger than that of primary amine hydrogen in ethylenediamine.

On the basis of these quantitative data, we can suppose that, in the very early stage of the curing reaction in the system DGEBA- $N$-methylethylenediamine, mostly the addition reaction of secondary hydrogen of $N$ methylethylenediamine to the epoxide ring takes place. The dependence of secondary amine group concentration on reaction time does not show a maximum (Fig. $4 \mathrm{a}, 40^{\circ} \mathrm{C}$ ), as is usual for consecutive reactions. In Fig. $4 \mathrm{~b}$ (curing at $60^{\circ} \mathrm{C}$ ), it is clearly seen that addition of primary amine hydrogen to the epoxide ring takes place simultaneously with reaction of the secondary $\mathrm{N}$-methylamino group of $\mathrm{N}$-methylethylenediamine. The shoulder on the secondary amine 
concentration curve demonstrates superposition of a decaying concentration of $\mathrm{N}$-methylamino groups with increasing concentration of secondary amine formed by reaction of primary amino groups with epoxide groups. This secondary amine is consumed in consecutive reactions with epoxy groups, giving tertiary amino groups.

\section{Fluorescence Monitoring of the Curing Reaction}

There have been substantial advances in the use of molecular probe spectroscopy for investigating mechanisms associated with complex relaxation behavior in amorphous polymer systems [15], solution properties of linear polymers $26-28$, polyelectrolytes $\sim$ 29-30, and polymer gelis 31-33.

The dansyl group has been widely used as a fluorescence label to study conformational transitions in proteins and synthetic polymers $26-30$. This group has a special photophysical property that gives information about the local hydrophilic-hydrophobic interactions and mobility of the microenvironment, as well as the binding properties of the group. Dansyl is thought to experience significant twisted intramolecular charge transfer (TICT) character in the ' $\mathrm{L}_{\mathrm{a}}$ excited state as the lone-pair electron on the amino group transfers into the $\pi^{*}$-orbital of the naphthalene ring. This charge transfer is enhanced by the electron-withdrawing sulfonyl group, resulting in the relatively large excited-state dipole moment $34-35$. The picture may be complicated by mixing of the ${ }^{\prime} \mathrm{L}_{a}$ charge transfer state and the ${ }^{\prime} \mathrm{L}_{b}$ state, which is dependent on solvent polarity, resulting in inversion of these energy levels [35]. Ghiggino et al. ̃ [34] observed that the TICT efficiency depends on solvent viscosity and coherence, such that strongly structured polar solvents would hinder TICT by sterically hindering conformational reorientations of the dimethylamino substituent. It is supposed that the shift of the emission maximum from 430 to $580 \mathrm{~nm}$ is determined by the twisting angle and speed of the dimethylamino group rotation with respect to the naphthyl group, which is affected by local interactions, viscosity, and free volume. The fluorescence quantum yield for dansyl derivatives ranges from less than 0.7 (hydrocarbon solvents) to 0.068 (water); the reasons for this variation are complex and only partly understood [32], [35].

In our case, the dansyl fluorophore was used as a label and/or probe for fluorescence monitoring of the curing reaction of diepoxide with primary amine and diamine. Generally, the curing reaction of diepoxides with amines or diamines is a polyaddition reaction characterized by stepwise increase in the molecular weight and changes in molecular weight distribution of the resulting products. Depending on the functionality of particular components, the increase in molecular weight is accompanied by branching and finally by cross-linking. Therefore, the curing reaction is accompanied by complex changes in all possible physicochemical properties of the system (hydrophilichydrophobic interactions, viscosity, dielectric constant, density, etc.).

The photophysical parameters of dansyl fluorophore that change during the curing process are fluorescence intensity and position of emission maximum and its half-bandwidth. These characteristics were evaluated depending on the cure time and on the epoxy group conversion in the system containing the DNS label and/or DNS probe. Owing to 
uncertainties in determination of the position of the emission maxima for broad emission bands of the DNS fluorophore encountered in our experimental data, the average value of the emission band position was evaluated and correlated with the degree of the epoxy group conversion as well.

\section{DGEBA- $n$-Butylamine}

The integrated fluorescence intensity of DNS fluorophore attached to the epoxide component in the curing system DGEBA- $n$-butylamine shows, at the beginning of the curing process, a surprisingly very small change (Fig. $1 \mathrm{c}$ ). In spite of the expected increase in the viscosity of the reaction mixture in this region, the integrated fluorescence intensity remains almost constant. To explain this observation, we can speculate that the fluorescence response of the DNS label reflects the behavior of low molecular weight species on the microscopic level. The DNS fluorophore attached to low molecular weight reaction products of DGEBA and $n$-butylamine reflects only small changes in the microenvironment of the DNS fluorophore. The rotational and translational diffusion of the DNS fluorophore caused by micro-Brownian motion is not obviously very much influenced by small changes in structure and molecular weight of the reaction intermediates in the early stage of the reaction (for epoxy group conversion, $\alpha<0.52$ ). The rotational and translational diffusion of the fluorophore influences the rate of nonradiative internal conversion processes and consequently the fluorescence quantum yield. A change in the macroscopic viscosity of the reaction mixture does not greatly affect the rotational and translational diffusion of the DNS label already limited by attachment to the low molecular weight species. Decrease in the mobility of DNS fluorophore takes place at epoxy group conversion higher than 0.52 caused by the abrupt increase in the molecular weight of linear polymer and increasing rigidity of the system.

A substantially different response in fluorescence of the DNS fluorophore was observed when this fluorophore was used as a probe (Figs. 1c and 2c). The integrated fluorescence intensity of the DNS probe increases from the beginning of the curing reaction and perhaps monitors the onset of the tertiary group concentration. After $100 \mathrm{~min}$ at $40^{\circ} \mathrm{C}$, the integrated fluorescence intensity reaches a plateau and increases sluggishly thereafter with curing time. The course of this dependence suggests that the DNS probe monitors the macroscopic viscosity of the reaction mixture. In this case, the rotational and translational mobility of the DNS probe at the beginning of the curing reaction is surely much higher than that of the DNS label. Even a small increase in the viscosity of the surrounding medium brings about remarkable changes in rotational and translational diffusion and, consequently, an increase in fluorescence quantum yield.

In Fig. 5 are shown other photophysical parameters for the DNS label and DNS probe in a stoichiometric reaction mixture of DGEBA- $n$-butylamine. Following our previous discussion, one can clearly see that the changes in these parameters are consistent with the course of the dependence of integrated fluorescence intensity on the epoxy group conversion. The fluorescence emission maxima represent an average of the microenvironments of probe and label in 
the reaction medium. The position of the emission maximum of the DNS label ( $20,300 \mathrm{~cm}$ ) at low conversion $(\alpha \rightarrow 0)$ clearly demonstrates partially limited label relaxation taking place in the side chain of the DGEBA dimer molecule in comparison with the DNS probe $\left(19,700 \mathrm{~cm}^{-1}\right)$. With increasing conversion, the microenvironment of the DNS label acts as a buffer, and a blue shift in the emission maximum was observed at higher conversions $(\alpha>$ $0.2-0.3)$.

Figure 5. The dependence of (a) the emission maximum (EM) and (b) half-bandwidth (HBW) on the epoxy group conversion $\alpha$ for the DNS label and probe in the stoichiometric reaction mixture DGEBA- $n$-butylamine. Curing temperature $40^{\circ} \mathrm{C}$.

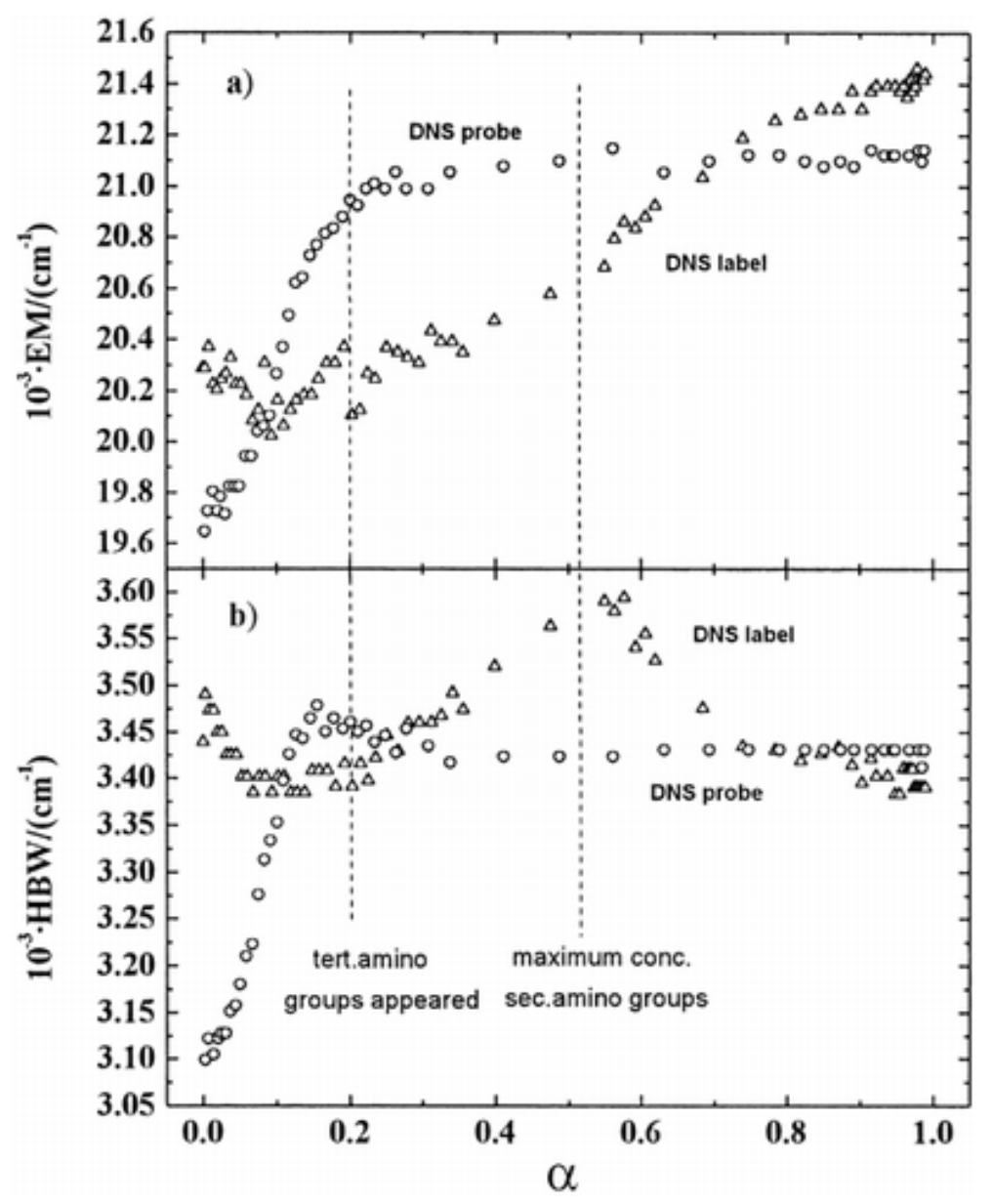

At the same time, the DNS label, in comparison with the DNS probe, monitors at low conversions a much broader spectrum of different interactions, including the DGEBA dimer microenvironment. In the dependence of label halfbandwidth on epoxy group conversion, the appearance of tertiary amino groups and the maximum of secondary amino group concentration in the reaction mixture is clearly indicated.

\section{DGEBA- $N$-Methylethylenediamine}

In Fig. 6 is shown, for illustration, the time evolution of the emission spectrum for the aforementioned system. The 
initial fast increase in the emission intensity of the DNS label is primarily due to the fast reaction of the secondary amino groups of $\mathrm{N}$-methylethylenediamine with epoxy groups, followed by a decrease in the emission intensity at the maximum but, at the same time, an enormous increase in the half-bandwidth.

Figure 6. Time evolution of the emission spectrum for the DNS-labeled stoichiometric reaction mixture DGEBA-Nmethylethylenediamine. Curing temperature $40^{\circ} \mathrm{C}$.

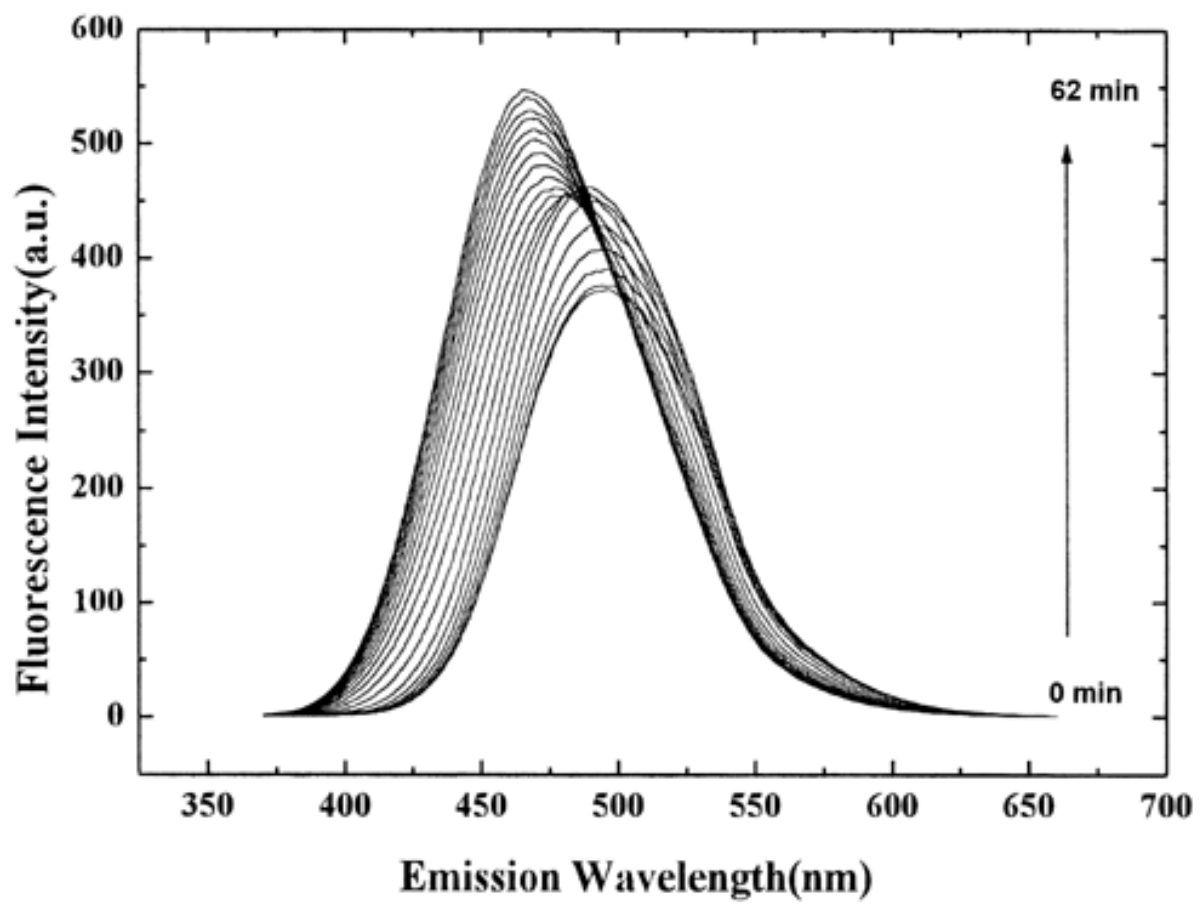

The change in integrated fluorescence intensity characterizing the curing process for the stoichiometric reaction mixture DGEBA- $N$-methylethylenediamine labeled with DNS and/or DNS probe, together with the dependence of the glass transition temperature on epoxy group conversion, is shown in Fig. 7. In the dependence of the integrated fluorescence intensity for the DNS label on the epoxy group conversion, one can observe several regions, as in the system DGEBA/ethylenediamine [17]:

Region 1. Reaction of epoxy groups mostly with secondary amino groups of $N$-methylethylenediamine is accompanied by an increase in the viscosity of the medium and a corresponding increase in fluorescence quantum yield of the DNS fluorophore attached to polymer chains. At the beginning of the curing reaction, tertiary amino groups arise solely by reaction of secondary amino groups of $\mathrm{N}$-methylethylenediamine with epoxy groups. Linear low molecular weight compounds are being formed. At the same time, a moderate increase in the half-bandwidth of the emission band can be observed (Fig. 8b).

Figure 7. The dependence of (a) the glass transition temperature $T_{\mathrm{g}}$, and integrated fluorescence intensity (Integ. Fluor. Int.) for (b) the DNS label and (c) DNS probe on the epoxy groups conversion $\alpha$ for the stoichiometric reaction mixture DGEBE- $N$-methylethylenediamine. Curing temperature $40^{\circ} \mathrm{C}$ for Figs. $7 \mathrm{~b}$ and 


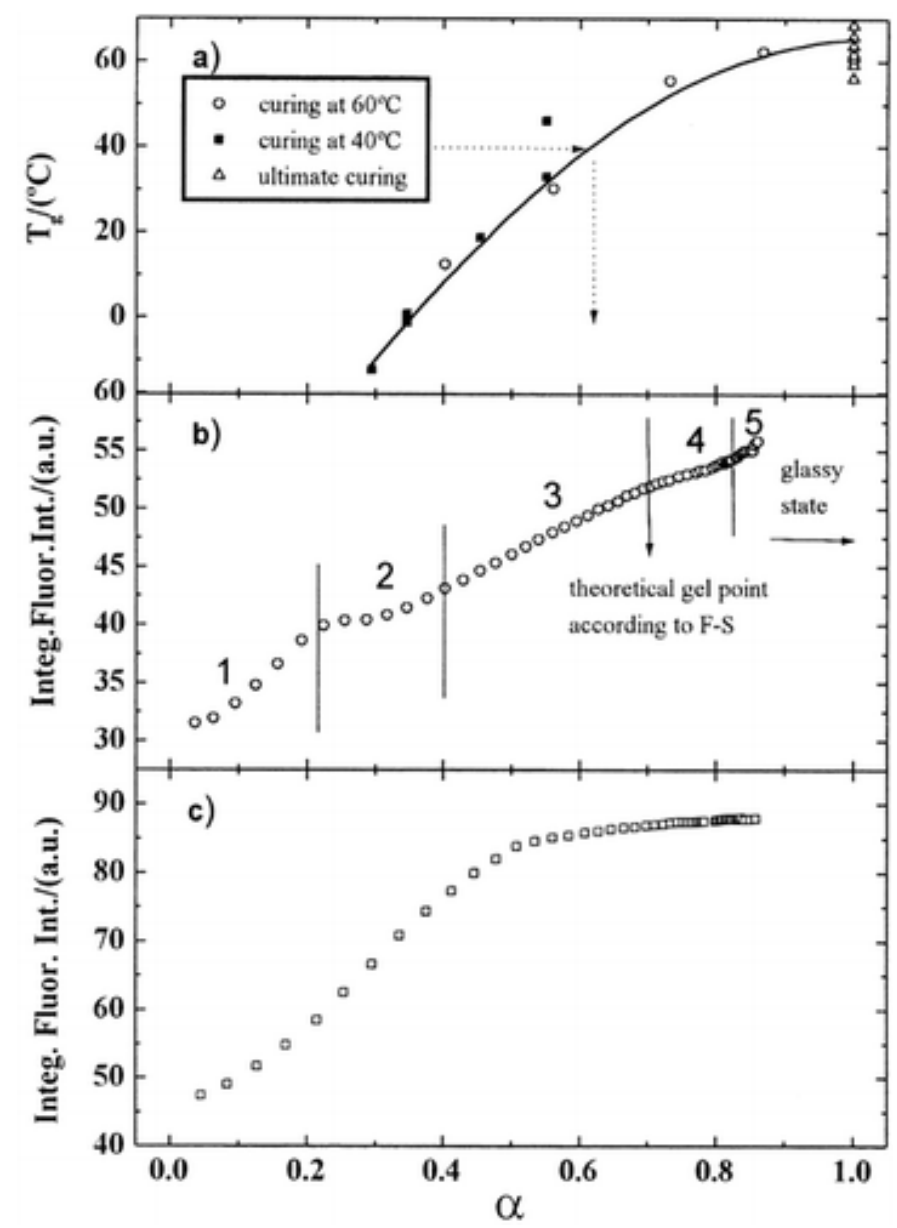

Figure 8. The dependence of (a) the position of the emission maximum (EM) and (b) half-bandwidth (HBW) for the DNS label and DNS probe on the epoxy groups conversion $\alpha$ in the stoichiometric reaction mixture DGEBA- $n$-butylamine. Curing temperature $40^{\circ} \mathrm{C}$. 


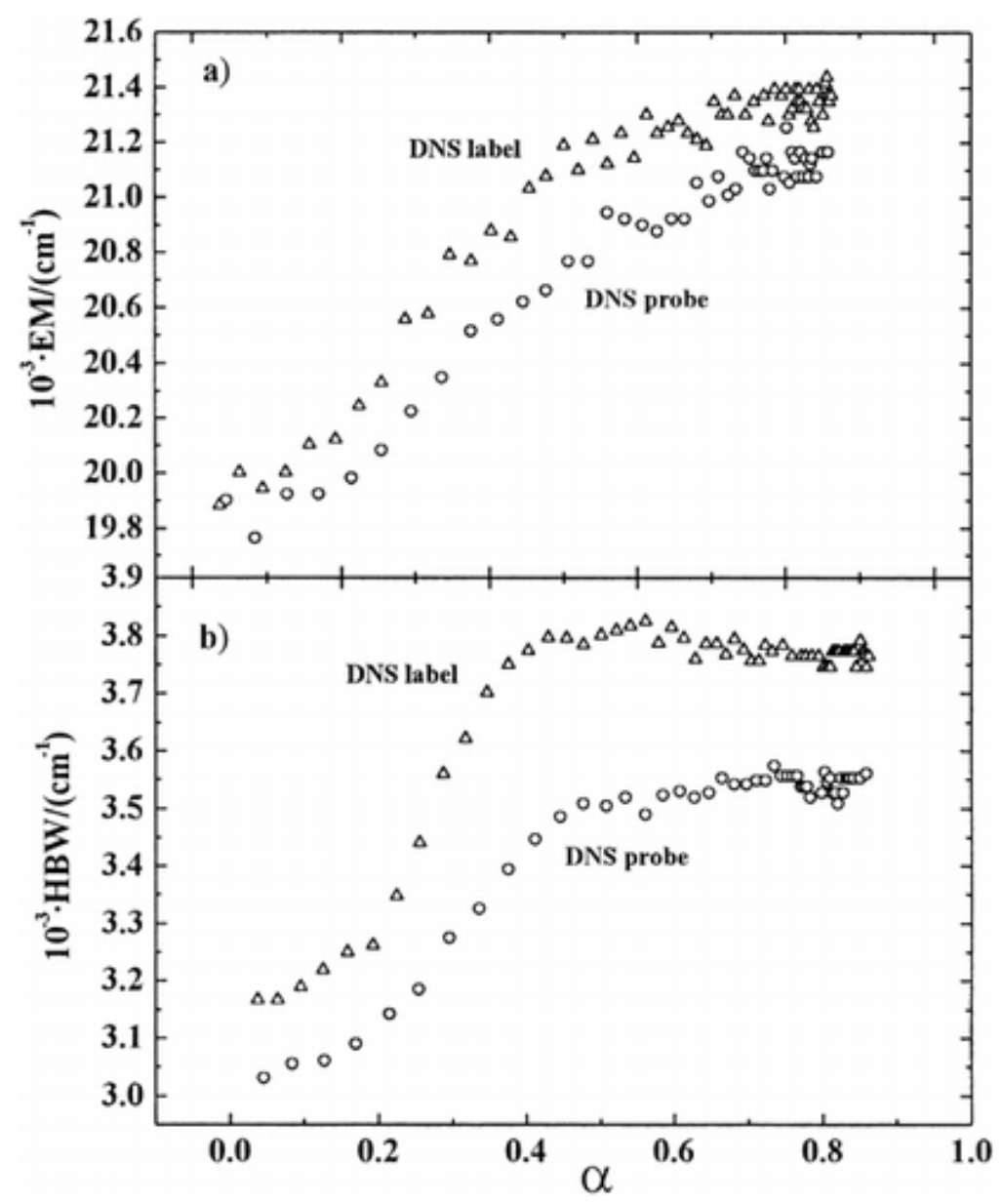

Region 2. An abrupt increase in the half-bandwidth with conversion takes place. We can assume that the large increase in half-bandwidth of the emission band is caused by the appearance of tertiary amino groups, the actual branching points, formed by reaction of secondary amino groups (mono- and diaddition products) with epoxide oligomers.

Region 3. A continuous increase in integrated fluorescence intensity takes place, caused by the increasing viscosity of the system. In this region, the molecular weight of polymer molecules increases by stepwise addition and branching reactions. At the epoxy group conversion $\alpha \quad 0.63$ (Fig. 7a), the glass transition temperature reaches the cure temperature $\left(40^{\circ} \mathrm{C}\right)$. The entry of the system into the vitrification region [37] is not monitored by either label or probe.

Region 4. The onset of this part of the curve is characterized by a change in slope that clearly shows the gel point (according to the Flory-Stockmayer theory, conversion of epoxy groups at the gel point for this system is 0.707). The increasing fraction of the gel in this region is accompanied by a moderate decrease in mobility of the DNS label and thereby an increase in its fluorescence quantum yield.

Region 5. A further steep increase in integrated fluorescence intensity at the epoxy group conversion $\alpha \quad 0.83$ was observed. According to analogy with other systems studied (kinetics and fluorescence study on curing 
DGEBA-ethylenediamine and/or $N, N^{\prime}$-dimethylethylenediamine) [17], we can interpret this change in the integrated fluorescence intensity as a consequence of entry of the system into the boundary between vitrifying and vitrified regions. On the threshold of the glassy state, strongly limited mobility of the polymer molecule segments imposes further mobility restriction on fluorophores attached to polymer molecules. As a consequence, an increase in the fluorescence quantum yield, and therefore in integrated fluorescence intensity, occurred.

The "wavy" course of fluorescence intensity is only observed when the fluorophore is attached to the polymer chain. The gel point and the last stage of curing reaction, which is characterized by entry of the system into the glassy state, are not sensed if the fluorophore is a probe (Fig. 7c). Similar to the DGEBA- $n$-butylamine stoichiometric reaction mixture, the emission of the DNS label in DGEBA- $N$-methylethylenediamine occurs from a less-relaxed fluorophore in comparison with the DNS probe. The half-bandwidth indicates a less homogeneous microenvironment for the DNS label than the probe in accordance with the generally accepted concept of the microenvironment of polymers and biological macromolecules in solutions.

\section{Monitoring of the Curing Process: Treatment of Experimental Data}

The change in fluorescence parameters of some fluorophores that accompanies polymerization reactions has been utilized for monitoring polymerization processes. According to the aforementioned results, the most significant features of some fluorophores is that they display a fluorescence wavelength shift and change in magnitude of fluorescence intensity as the polymerization reaction proceeds.

The run-to-run reproducibility of the profile shape of the fluorescence intensity signal is generally good; however, the reproducibility of the absolute intensity values when measuring samples in front-face geometry is, in most cases, unsatisfactory. For this reason, an intensity ratio method using fluorophores for monitoring polymerization processes has been reported [38].

Changes in the fluorescence maximum wavelength as a function of cure degree produce a highly characteristic signal profile that is reproducible and also reveals, sometimes, the main chemorheological events with a distinct change in slope. However, the determination of the emission maximum for broad emission bands (e.g., DNS fluorophore) is not very accurate and suffers from large scatter. We have proposed to evaluate the average value of the emission band position $<v>$ defined by Eq. 7 :

$$
<\mathrm{V}\rangle=\Sigma I_{F}(\mathrm{v}) \mathrm{v} / \Sigma I_{F}(\mathrm{v})
$$

where $I_{F}(v)$ is the intensity of fluorescence at wavenumber $v$. In Figs. $9 \mathrm{a}$ and $9 \mathrm{~b}$ is shown, for comparison, the dependence of the emission maximum and $\langle v>$ on epoxy group conversion for DNS probe and DNS label in the stoichiometric reaction mixture DGEBA- $n$-butylamine. The same dependence for the DNS probe in DGEBA-Nmethylethylenediamine reaction mixture is depicted in Fig. 10. A smooth correlation was found in all cases between 
the average value of the emission band position $\langle v\rangle$ and the degree of epoxy group conversion in comparison with the analogous dependence for the emission maximum. The results show that correlation between $<v>$ and conversion degree provides a method for monitoring the curing of epoxide systems. A further study on curing of different epoxide formulations and polymerization by the free-radical mechanism monitored by fluorescence is currently under preparation.

Figure 9. The dependence of the position of the emission maximum (EM) and the average value of emission band position $\langle v>$ on the epoxy group conversion $\alpha$ for the (a) DNS probe and (b) DNS label in the stoichiometric reaction mixture DGEBA- $n$-butylamine. Curing temperature $40^{\circ} \mathrm{C}$.

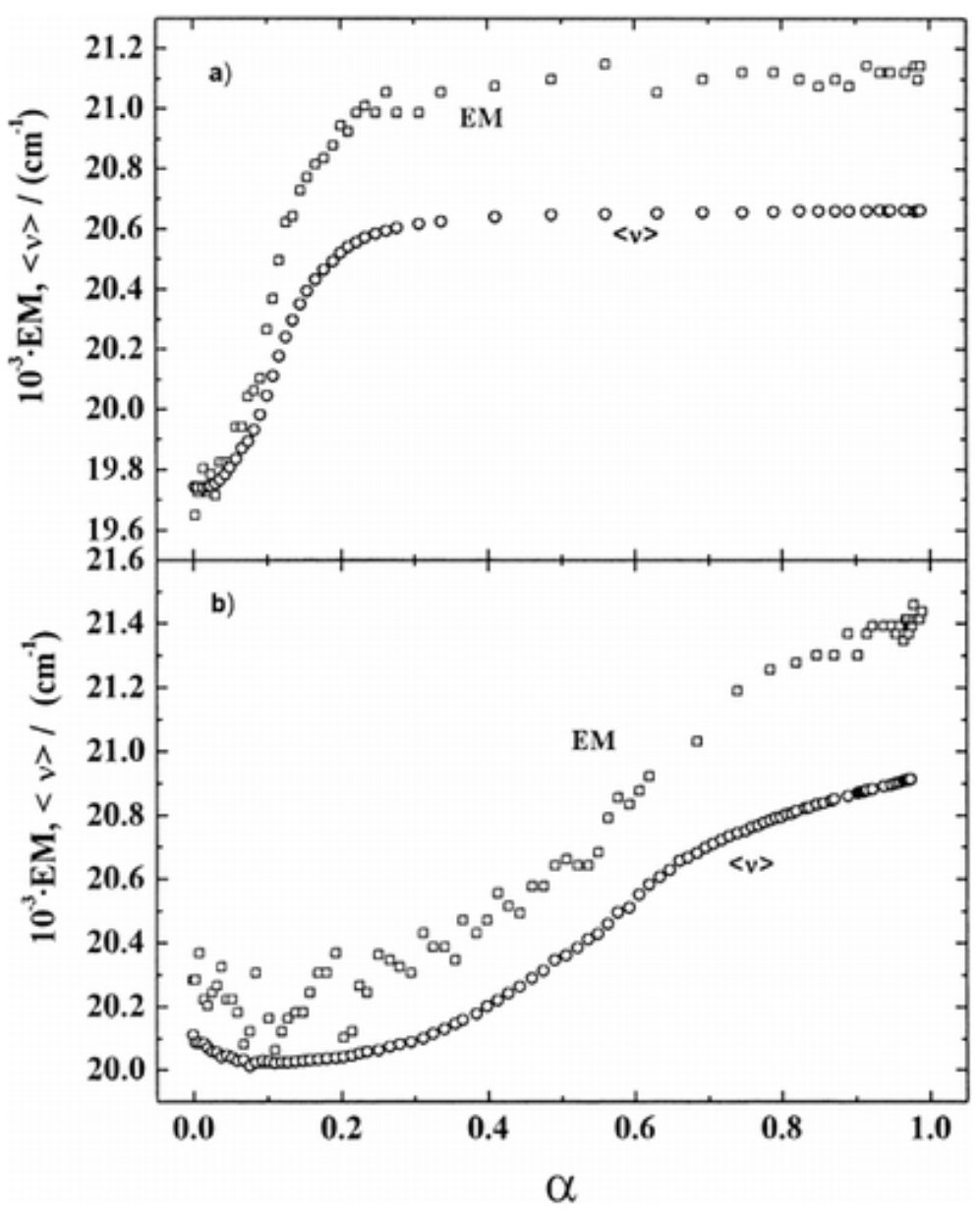

Figure 10. The dependence of the position of the emission maximum (EM) and the average value of emission band position $\langle v>$ on the epoxy group conversion $\alpha$ for the DNS probe in the stoichiometric reaction mixture DGEBA- $N$-methylethylenediamine. Curing temperature $40^{\circ} \mathrm{C}$. 


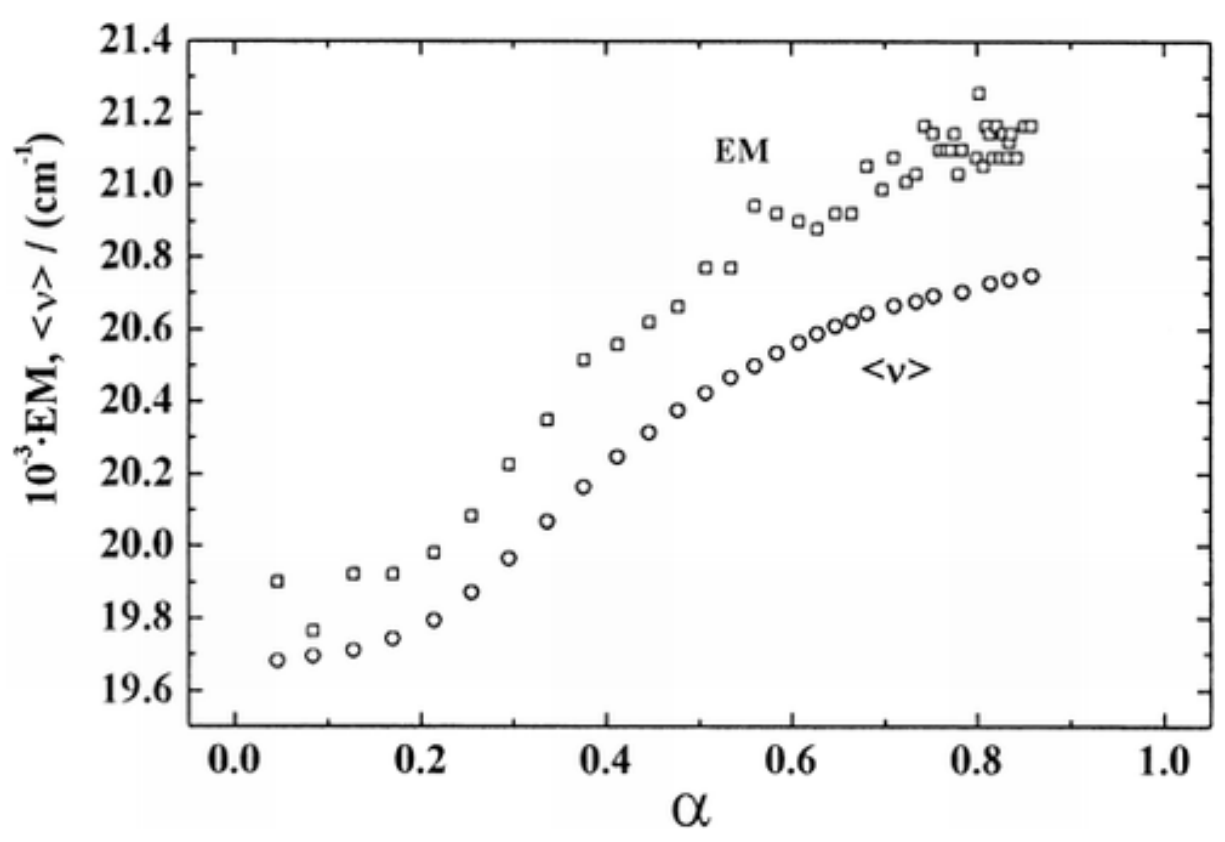

\section{SUMMARY AND CONCLUSIONS}

We interpret the evidence summarized in this article as indicating that the stoichiometric reaction mixture of DGEBA with $n$-butylamine at $\alpha \quad 0.52$ consists of mono- and diaddition products arising by addition of the primary amino hydrogen to the epoxy groups of DGEBA. At higher conversion of the epoxy groups $(\alpha>0.52)$, further increase in molecular weight of the reaction product takes place by a stepwise addition reaction.

The course of the dependence of integrated fluorescence intensity on cure time for the DNS-labeled reaction mixture DGEBA- $n$-butylamine follows the most important chemical transformations occurring in the reaction mixture, that is, the onset of tertiary amino group concentration and the maximum concentration of secondary amino groups in the reaction mixture.

Changes in integrated fluorescence intensity for the DNS probe in the DGEBA- $n$-butylamine reaction mixture indicate the onset of tertiary amino group concentration only. The studies of fluorescence (integrated fluorescence intensity, emission maximum, half-bandwidth) establish that the DNS label groups experience a microenvironment of DGEBA dimer and oligomers in comparison with the DNS probe.

Higher reactivity of the secondary amino group hydrogen in $\mathrm{N}$-methylethylenediamine than that of the primary amino group gives rise to a fast increase in concentration of tertiary amino groups $\left(\mathrm{CH}_{3} \quad \mathrm{~N}\right.$ ) in the DGEBA-Nmethylethylenediamine reaction mixture. Later, a further increase in molecular weight of the reaction product is caused by stepwise addition, branching reactions, and finally cross-linking. 
The integrated fluorescence intensity for the DNS-labeled reaction mixture mentioned above indicates important physicochemical transformations occurring in the system - the gel point and entry of the system into the boundary between the rubbery and glassy states. The DNS probe does not sense any of these transformations.

The correlation between the average value of the emission band position $<v>$ for both the DNS label and the DNS probe and the epoxy group conversion can be used to evaluate the degree of epoxy group conversion in epoxide systems in real time and in situ. The following two points outline the remaining and continuing uncertainties for the particular curing systems.

1. The major uncertainty in the interpretation of these data concerns the question of the very different fluorescence responses of DNS fluorophores (probe vs. label) in the system DGEBA- $n$-butylamine. These findings, obtained by a steady-state fluorescence method, are now being addressed by time-resolved fluorescence spectroscopy to determine rotational relaxation times of DNS fluorophore (label and/or probe) in neat DGEBA and in partially cured systems by $n$-butylamine and/or $N$-methylethylenediamine and to reconcile these observations.

2. We also wish to describe more exactly the first half (wavy course) of the dependence of integrated intensity on epoxy group conversion (DGEBA-N-methylethylenediamine). This task requires resolution of the overall concentration of secondary and tertiary amino groups into particular contributions.

\section{ACKNOWLEDGMENT}

We would like to thank the European Commission for funding through the BRITE-EuRam Project (BE97-4472) and to $\mathrm{CAM}(07 \mathrm{~N} / 0002 / 98)$.

\section{REFERENCES}

1. Sung, C. S.P. , Pyun, E. and Sung, H. L. 1986. Characterization of Epoxy Cure by UV-Visible and Fluorescence Spectroscopy: Azochromophoric Labeling Approach. Macromolecules, 19: 2922 -2932 . [Google Scholar]

2. Levy, R. L. and Schwab, S. D. 1988 . "Performance Characteristics of the Fluorescence Optrode Cure Sensor". In Cross-linked Polymers, ACS Symposium Series 367 Edited by: Dickie, R. A., Laban, S. S. and Bauer, R. S. 113-121. Washington, DC : American Chemical Society . 
3. Wang, F. W., Lowry, R. E. and Fabconi, B. M. 1986. Novel Fluorescence Method for Cure Monitoring of Epoxy Resins. Polymer, 27: 1529

4. Levy, R. L. and Ames, D. P. 1984 . Monitoring Epoxy Cure Kinetics with a Viscosity-Dependent Fluorescence Probe. Polym. Sci. Technol., 29: 245-256.

5. Sung, C. S.P. , Chin, I.-J. and Yu, W.-Ch. 1985 . A Novel Fluorescence Technique for Monitoring Cure Reactions in Epoxy Networks. Macromolecules, 18: 1510-1512 .

6. Sung, C. S.P., Pyun, E. and Sun, H.-L. 1986. Characterization of Epoxy Cure by UV-Visible and Fluorescence Spectroscopy: Azochromophoric Labeling Approach. Macromolecules, 19: 2922 -2932 .

7. Yu, W.-C. and Sung, C. S.P. 1990 . Excitation Spectroscopy of a Reactive Label for Characterization of the Cure Process in an Epoxy Network. Macromolecules, 23: 386-390 .

8. Pyun, E. and Sung, C. S.P. 1991 . Network Structure in Diamine-Cured Tetrafunctional Epoxy by UV-VIS and Fluorescence Spectroscopy. Macromolecules, 24: 855-861.

9. Song, J. C. and Sung, C. S.P. 1993 . Fluorescence Studies of Diaminodiphenyl Sulfone Curing Agent for Epoxy Cure Characterization. Macromolecules, 26: $4818-4824$.

10. Paik, H.-J. and Sung, N.-H. 1994 . Fiberoptic Intrinsic Fluorescence for In-Situ Cure Monitoring of Amine Cured Epoxy and Composites. Polym. Eng. Sci., 34(12): 1025 -1032 .

11. Strehmel, B., Strehmel, V. and Younes, M. 1999. Fluorescence Probes for Investigation of Epoxy Systems and Monitoring of Crosslinking Processes. J. Polym. Sci., Part B: Polym. Phys., 37: 1367 -1386 .

12. Reichardt, C. 1994 . Solvatochromic Dyes as Solvent Polarity Indicators . Chem. Rev., 94: 2319-2358 .

13. Paley, M. S., McGill, R. A., Howard, S. C., Wallace, S. E. and Harris, J. M. 1990 . Solvatochromism. A New Method for Polymer Characterization. Macromolecules, 23: 4557 -4564. 
14. Loufty, R. O. and Teegarden, D. M. 1983 . Effect of Polymer Tacticity on the Fluorescence of Molecular Rotors. Macromolecules, 16:452-456.

15. Ediger, M. D. 1991 . Time-Resolved Optical Studies of Local Polymer Dynamics. Annu. Rev. Phys. Chem., 42: $225-250$.

16. Valdes-Aquilera, O., Pathak, C. P. and Neckers, D. S. 1990 . Pyrene as a Fluorescent Probe for Monitoring Polymerization Rates. Macromolecules, 23: 689-692.

17. Mikeš, F., González-Benito, F. J. and Baselga Llidó, J. in press . Macromol.,

18. Song, J. C., Torres-Filho, A. and Neckers, D. C. Intramolecular Charge Transfer Complexes as Fluorescence Probes for UV and visible light induced acrylate Polymerization. Rad. Tech'94 North Am. UV/EB Conf. Exhib. Proc. Vol. 1, pp.338 -343. Northbrook, IL : Rad. Tech. Int. North Am.

19. Morgan, R. J. 1985. Structure-Property Relations of Epoxies Used as Composite Matrices. Adv. Polym. Sci., 72: $1-44$.

20. George, G. A., Cole-Clarke, P., St John, N. A. and Friend, G. 1991 . Real-Time Monitoring of the Cure Reaction of a TGDDM/DDS Epoxy Resin Using Fiber Optic FT-IR. J. Appl. Polym. Sci., 42: 643-657.

21. St. John, N. A. and George, G. A. 1992 . Cure Kinetics and Mechanism of a Tetraglycidyl-4',4diaminodiphenylmethane/diaminidiphenylsulphone Epoxy Resin Using Near IR Spectroscopy. Polymer, 33(13): $2679-2688$

22. Leezenberg, P. B. and Frank, C. W. 1995 . Selective Sorption and Solvatation of Dansyl-Labeled Poly(dimethylsiloxane) Networks Swollen in Binary Solvent. Macromolecules, 28: 7407 -7415 .

23. Eisinger, J. and Flores, J. 1979 . Front-Face Fluorometry of Liquid Samples. Anal. Biochem., 94: 15-21.

24. Horie, K., Hiura, H., Sawada, M., Mita, I. and Kambe, H. 1970 . Calorimetric Investigation of Polymerization Reactions. III. Curing Reaction of Epoxides with Amines. J. Polym. Sci. A-1, 8: 1357-1372 . 
25. Paz-Abuin, S., Lopez-Quintela, A., Varela, M., Pazos-Pellin, M. and Prendes, P. 1997 . Method for Determination of the Ratio of Rate Constants, Secondary to Primary Amine, in Epoxy-Amine Systems. Polymer, 38(12): $3117-3120$.

26. Chen, H. and Morawetz, H. 1982 . Kinetics of Polymer Complex Interchange in Poly(acrylic Acid)Poly(oxyethylene) Solutions. Macromolecules, 15: 1445-1447.

27. Winnik, F. M. 1990 . Fluorescence Studies of Aqueous Solutions of Poly( $\quad N$-isopropylacryl-amide) Below and Above LCST. Macromolecules, 23: 233-242.

28. Binkert, Th., Oberreich, J., Meewes, M., Nyffenegger, R. and Ricka, J. 1991 . Coil-Globule Transition of Poly(N-isopropylacrylamide): A Study of Segment Mobility by Fluorescence Depolarization. Macromolecules, 24: $5806-5810$.

29. Strauss, U. P. and Vesnaver, G. 1975 . Optical Probes in Polyelectrolyte Studies. II. Fluorescence Spectra of Dansylated Copolymers of Maleic Anhydride with Alkyl Vinyl Ethers. J. Phys. Chem., 79: 2426 -2439 .

30. Bednár, B., Trnená, J., Svoboda, P., Vajda, Š, Fidler, V. and Procházka, K. 1991. Time-Resolved Study of Chain Dynamics. 1. Poly(methacrylic Acid) in Dilute Water Solutions. Macromolecules, 24: 2054 -2059 .

31. Shea, K. J., Sasaki, D. Y. and Stoddard, G. J. 1989 . Fluorescence Probes for Evaluating Chain Solvatation in Network Polymers. An Analysis of the Solvatochromic Shift of the Dansyl Probe in Macroporous StyreneDivinylbenzene and Styrene-Diisopropenylbenzene Copolymers. Macromolecules, 22: 1722 -1730 .

32. Shea, K. J., Okahata, Y. and Doughert, T. K. 1984 . Fluorescence Probes in Polymer Chemistry. Application of 5(Dimethylamino)-1-Naphthalenesulfonamides to the Study of Solvatation of Styrene-Divinylbenzene Copolymers. Macromolecules, 17: 296-300.

33. Hu, Y., Horie, K. and Usiki, H. 1992 . Fluorescence Studies of the Volume Phase Transition of Poly(acrylamide) Gels with a Dansyl Group. Macromolecules, 25: 6040 -6044 .

34. Ghiggino, K. P., Lee, A. G., Meech, S. R., O'Connor, D. V. and Phillips, D. 1981 . Time Resolved Emission 
Spectroscopy of the Dansyl Fluorescence Probe. Biochemistry, 20: $5381-5389$.

35. Li, Y.-H., Chan, L.-M., Tyer, L., Moody, R. T., Himel, C. M. and Hercules, D. M. 1975 . Study of Solvent Effects on the Fluorescence of 1-(Dimethylamino)-5-Naphthalenesulfonic Acid and Related Compounds. J. Am. Chem. Soc., 97: $3115-3126$.

36. Kosower, E. M., Dodiuk, H., Tazutake, K., Ottolenghi, M. and Orbach, N. 1975 . Intramolecular Donor-Acceptor Systems. Radiative and Nonradiative Processes for the Excited States of 2-N-Arylamino-6naphthalenesulfonates. J. Am. Chem. Soc., 97: 2167-2178.

37. Verchère, D., Sautereau, H., Pascault, J. P., Riccardi, C. C., Moschiar, S. M. and Williams, R. J.J. 1990 . Buildup of Epoxycycloaliphatic Amine Networks. Kinetics, Vitrification, and Gelation. Macromolecules, 23: 725 -731 .

38. Song, J. C. and Neckers, D. C. 1996 . Characterization of Photocurable Coatings Using Fluorescence Probes. Polym. Eng. Sci., 36(3): 394-402 . 